Physics, Chemistry, and Dynamics of Interplanetary Dust

ASP Conference Series, Vol. 104, 1996

Bo A. S. Gustafson and Martha S. Hanner (eds.)

\title{
Fragmenting Particles During 1989-1994 Perseid Radar Observations
}

\author{
M. Šimek \\ Astronomical Institute of the Czech Academy of Sciences, \\ 25165 Ondrejov, Czech Republic
}

\begin{abstract}
The occurrence of fragmenting particles was investigated from radar observations of the Perseids at Ondrejov Observatory. Diurnal patterns of fragmenting meteoroids are presented. Their minimum occurrence coincides with the culmination of the Perseid radiant while the maximum appears at the time of sunset at meteor heights. Solar longitude of the main contribution of fragmenting meteoroids at $L_{\odot}=138^{\circ} .86 \pm 0^{\circ} .04$ (equinox 1950.0) fits with the peak activity of the new filament in the stream. This supports the concept of the low age of the filament. The maximum proportion of fragmenting particles occurred in 1991.
\end{abstract}

\section{Introduction}

It was found that radio echoes indicating fragmenting particles appeared more frequently during Perseid observations in the past few years than before (Šimek, 1993a). Fragmenting particles are characterized on radar records by a multicomplex. structure of radio-echo reflections along a meteor trajectory in the Earth's atmosphere when single fragments of a meteor particle produce a complex of ionized trails practically parallel to the main trail, and more points then fulfill the condition of specular reflection at different ranges. Physical properties of fragmentation were analyzed by Cook (1954), Jacchia (1955), Jacchia et al. (1965), Verniani (1969), Bronshten (1983), and others. Kramer (1968) pointed out that some meteoroids of cometary origin contain inclusions which are sufficiently volatile to provide fragmentation of these particles not only in the Earth's atmosphere but also outside its limits. Such meteoroids disintegrate more easily and, as a consequence, they should be products of relatively recent ejections from the parent comet (Trigo, 1992; Šimek, 1993a). The nodal distribution of the Perseid meteor stream was analyzed by Harris et al. (1995). Watanabe (1993), Wu \& Williams (1993), Williams \& Wu (1994), Pecina \& Simek (1996), and others found the 1862 passage of $\mathrm{P} / \mathrm{Swift}$-Tuttle through its perihelion as the most appropriate place and time for such an ejection.

\section{Analysis}

Activity appearing from the new filament of the Perseids since 1986 near solar longitude $L_{\odot}=138^{\circ} .87$ (equinox 1950.0) has been analyzed by Šimek \& Pecina (1996). They found distinct activity variations of the new filament which culminated in 1992 and 1993. The present investigation of fragmenting particles covers the 1989-1994 period of radar observations at the Ondrejov Observatory $\left(14^{\circ} 47^{\prime} 1^{\prime \prime} \mathrm{E}, 49^{\circ} 54^{\prime} 38^{\prime \prime} \mathrm{N}\right)$. 
The proportion of echoes showing fragmentation in the combined number of both shower and sporadic echoes with duration $T \geq 0.4 \mathrm{~s}$ was chosen as a measure of the occurrence of the fragmenting particles. Its diurnal variation depends on the zenith distance $z$ of the Perseid radiant and on the mass distribution index $s$ of all observed meteors, which reflects the effect of the sunlit phase on observed meteor rates (Šimek, 1993b). The echo counts were therefore made in one-hour observing intervals corresponding to $\Delta L_{\odot}=0^{\circ} .04$. To cover the principal part of Perseid activity, the range of solar longitudes $137^{\circ} .64 \leq L_{\odot}<140^{\circ} .52(1950.0)$ was chosen for the analysis. Individual Perseid meteors cannot be directly selected from our records and, therefore, counted rates also contain sporadic meteors.

First, the investigated range of $L_{\odot}$ was divided into three parts each of them covering a 24 hour interval. The results are summarized in Table ?? which contains the distribution of the ratio of fragmenting to non-fragmenting particles having echo durations $T \geq 0.4 \mathrm{~s}$ in per cent for each individual year. Since whole 24 -h intervals were considered, the ratios of fragmenting particles are not affected by their diurnal variation resulting from changes of zenith distance of the shower radiant. The greatest portion of fragmenting particles occurred mostly in the central $L_{\odot}$ interval which was analyzed in more details (see below).

Table 1. The ratio of the number of fragmenting to non-fragmenting meteoroids having echo duration $T \geq 0.4 \mathrm{~s}$ in per cent in three $L_{\odot}$ intervals. $N$ designates number of fragmenting particles in sample.

\begin{tabular}{cccrrrr}
$N, L_{\odot} /$ year & $N$ & $137^{\circ} .64-138^{\circ} .60$ & $N$ & $138^{\circ} .60-139^{\circ} .56$ & $N$ & $1399^{\circ} .56-140^{\circ} .52$ \\
\hline 1989 & - & - & 20 & 5.9 & 10 & 7.6 \\
1990 & 26 & 4.0 & 49 & 7.6 & 17 & 9.1 \\
1991 & 19 & 3.7 & 136 & 16.4 & 31 & 7.7 \\
1992 & 72 & 5.6 & 165 & 11.5 & 60 & 10.1 \\
1993 & 69 & 7.6 & 131 & 10.5 & 84 & 8.7 \\
1994 & 45 & 6.6 & 82 & 9.2 & 32 & 4.6
\end{tabular}

Considering constant flux of fragmenting particles during the first and third $L_{\odot}$ intervals presented in Table ??, their mean percentages, $F$, in each particular hour were found. The gathered diurnal variation provided factors for correction of the diurnal variation of the fragmenting echo percentage in each hour (see Fig. ??). The contribution of fragmenting meteoroids is obviously controlled by the zenith angle of the shower radiant, particularly between $0 \mathrm{~h}$ and $16 \mathrm{~h}$ Local time. The patterns in Fig. ?? show the maximum at 20h LT, corresponding with the time of sunset at a meteor height of $100 \mathrm{~km}$ at $20 \mathrm{~h} 27 \mathrm{~m} \mathrm{LT}$. It seems that there are two superimposed effects, i.e. that of the radiant zenith distance $z$ combined with the number of daylight hours at the meteor heights. Proportion of fragmenting particles is also affected by lower counts of sporadic meteors in the evening hours. However, since their activity is in general considerably lower in comparison with shower echo rates, their effect would not be very significant. The abundance of $F$ is almost symmetrical and takes about six hours.

\section{Main contribution of fragmenting particles}

It was found that the highest number of fragmenting particles appeared within the range of $138^{\circ} .80 \leq L_{\odot}<138^{\circ} .96$. This solar longitude interval corresponds 


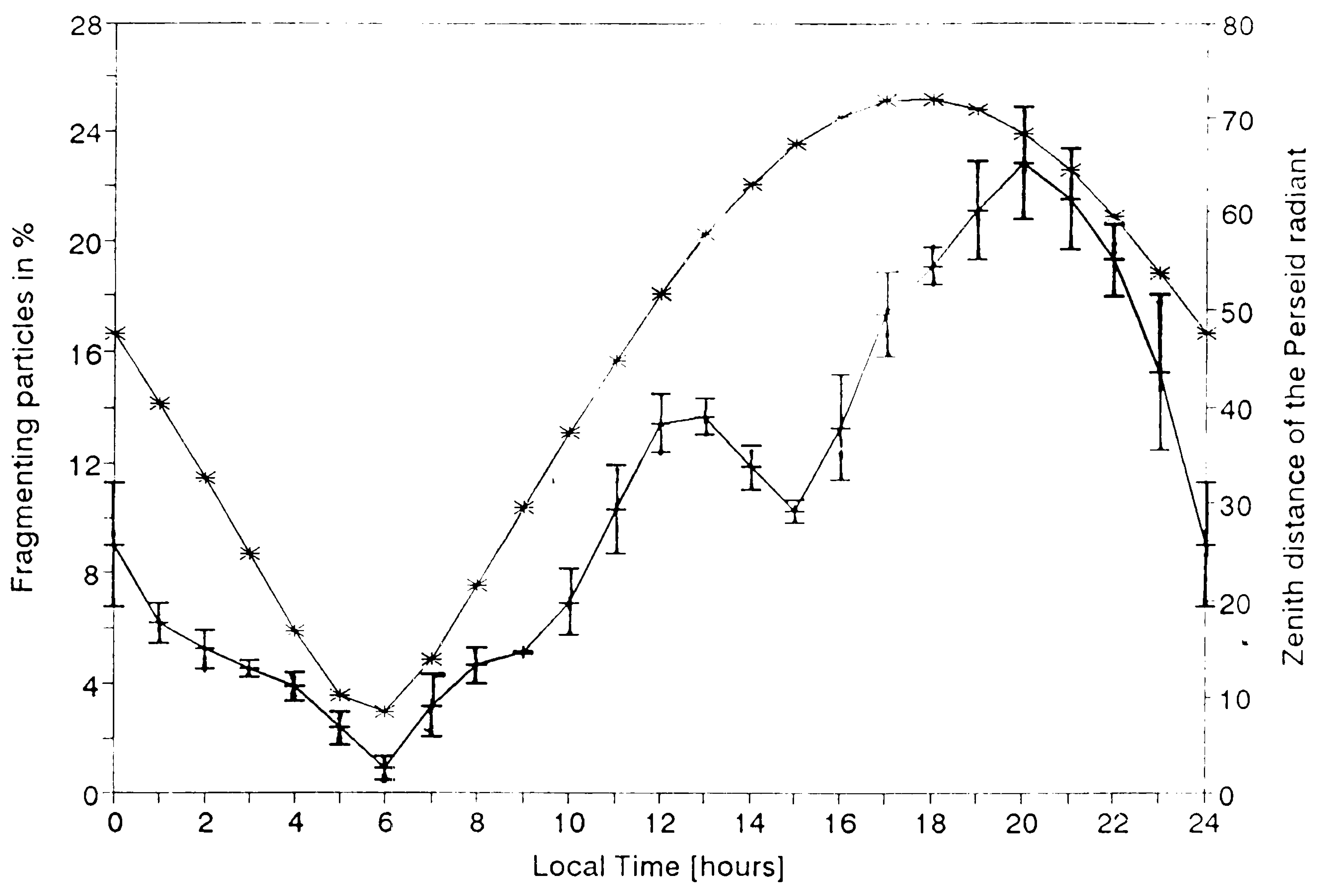

Figure 1. The ratio of fragmenting to non-fragmenting particles having echo duration $T \geq 0.4 \mathrm{~s}$ in per cent $(+)$. Error bars show the siandard deviation of the mean value in the sample. Zenith distance of the Perseid radiant is indicated *.

with different Local time periods and, therefore, the corrections discussed above were applied to produce the diurnal patterns of recorded fragmenting particles. The results are shown in Table ?? and Table ??. The center of fragmentation activity near $L_{\odot}=138^{\circ} .86$ fits perfectly with the location of the culmination of the meteor activity of the new filament determined by Šimek \& Pecina (1995).

Table 2. The distribution of fragmenting particles in per cent in one hour intervals in the range of solar longitudes $138^{\circ} .80 \leq L_{\odot}<138^{\circ} .96$. $138^{\circ} .80 \leq L_{\odot}<138^{\circ} .84 \quad 138^{\circ} .84 \leq L_{\odot}<138^{\circ} .88 \quad 138^{\circ} .88 \leq L_{\odot}<138^{\circ} .92 \quad 138^{\circ} .92 \leq L_{\odot}<138^{\circ} .96$

$\begin{array}{llll}14.2 \pm 3.0 & 14.4 \pm 4.3 & 10.2 \pm 2.5 & 9.8 \pm 2.0\end{array}$

Table 3. The distribution of percentage of fragmenting particles in different years in the range of solar longitudes $138^{\circ} .80 \leq L_{\odot}<138^{\circ} .96$.

\begin{tabular}{cccccc}
1989 & 1990 & 1991 & 1992 & 1993 & 1994 \\
\hline $7.9 \pm 3.7$ & $10.2 \pm 3.0$ & $21.9 \pm 4.4$ & $13.5 \pm 1.0$ & $7.6 \pm 2.4$ & $11.8 \pm 3.1$
\end{tabular}

The percentage of fragmenting particles in individual years from 1989 to 1994 are shown in Table ??. The large standard deviations permit cnly the conclusion that the ratio of fragmenting particles in 1989 and 1993 was considerably lower than in the other years. The maximum number of fragmenting particles occurred in 1991. 


\section{Conclusions}

The diurnal variation of fragmenting particles shows a minimum near the culmination of the Perseid radiant while the maximum fragmentation appears at the time of sunset at the height of $100 \mathrm{~km}$. The first part of the curve confirms the suggestion that fragmentation depends on the radiant zenith angle (Jacchia, 1955). The fragmentation is more frequent between $17 \mathrm{~h}$ and $23 \mathrm{~h}$ Local time indicating an effect of large zenith angle of relevant meteor trajectories in the Earth's atmosphere causing an anomalous ablation process possibly combined by the duration of the daylight at the meteor heights. The agreement of the position of the maximum fragmenting particles with the peak activity of the new filament in the Perseid meteor stream supports the idea of the greater fragility of those particles recently ejected from the parent comet during last few perihelion passages.

Acknowledgments. This research was supported by grant No 303104 of the Grant Agency of the Academy of Sciences of the Czech Republic. The author is indebted to two anonymous referees for valuable comments which improved the paper.

\section{References}

Bronshten, V. A. 1983, Physics of meteoric phenomena, Dordrecht: Reidel

Cook, A. F. 1954, ApJ, 120, 272

Harris, N. W., Yau, K. K. C., \& Hughes, D. W. 1995, MNRAS, 273, 999

Jacchia, L. G. 1955, ApJ, 121, 521

Jacchia, L. G. 1958, Smitsonian Contr. Astrophys., 2, 181

Jacchia, L. G., Verniani, F., \& Briggs, R. E. 1965, Smitsonian Contr. Astrophys., 10,1

Kramer, E. N. 1968, in Physics and dynamics of meteors, L.Kresk \& P. M. Millman, Dordrecht: Reidel, 236

Pecina, P., \& Šimek M. 1996, submitted to $A \& A$

Šimek M. 1993a, WGN, 21, 13

Šimek M. 1993b, in Meteoroids and their parent bodies, J. Štohl \& I. P. Williams, Astronomical Institute of the Slovak Academy of Sciences, Bratislava, 277

Šimek, M., \& Pecina, P. 1995 (this Colloquium)

Trigo, J. M. 1992, WGN, 20, 105

Verniani, F. 1969, Space Sci. Rev., 10, 230

Watanabe, J. 1993, in Meteoroids and their parent bodies, J. Štohl \& I. P. Williams, Astronomical Institute of the Slovak Academy of Sciences, Bratislava, 197

Williams, I. P., \& Wu, Z. 1994, MNRAS, 269, 524

Wu, Z., \& Williams, I. P. 1993, MNRAS, 264, 980 